\title{
ALMOST PERIODIC SOLUTIONS OF SECOND ORDER NONLINEAR DIFFERENTIAL EQUATIONS WITH ALMOST PERIODIC FORCING
}

\author{
C. E. LANGENHOP AND G. SEIFERT ${ }^{1}$
}

1. In this paper we give conditions for the existence of an almost periodic (a.p. for short) solution of the nonlinear differential equation $^{2}$

$$
x^{\prime \prime}+f(x) x^{\prime}+g(x)=k e(t)
$$

where $e(t)$ is a.p. and $|e(t)| \leqq 1$. Moreover, we establish a region of the $(x, y)$ plane in which this solution is unique and asymptotically stable with respect to other solutions in this region; here

$$
y=x^{\prime}+F(x), \text { where } F(x)=\int_{0}^{x} f(v) d v .
$$

We write (1.1) in the form of an equivalent system:

$$
\begin{aligned}
& x^{\prime}=y-F(x), \\
& y^{\prime}=-g(x)+k e(t),
\end{aligned}
$$

the $F(x)$ being as defined above. Our conditions on $g$ and $f$ are essentially as follows: $f(x) \geqq \alpha>0$ for some constant $\alpha, g(0)=0$, the derivative $g^{\prime}(x)$ is continuous for all $x$ in a certain interval containing the origin, and $g^{\prime}(x)>0$ for $x \neq 0$.

System (1.2) may be regarded as a special case $(n=2)$ of the vector equation:

$$
u^{\prime}=L u+r(u)+k q(t)
$$

where $u, r$, and $q$ are vectors in Euclidean $n$-space, $q(t)$ is a.p., $L$ is a constant $n \times n$ matrix, $r(0)=0$, and for each $\lambda>0$ there exists a $\delta>0$ such that $|u|<\delta,|v|<\delta$ imply $|r(u)-r(v)|<\lambda|u-v|$; here || denotes any norm in the vector space. In our case,

$$
L=\left(\begin{array}{ll}
-f(0) & 1 \\
-g^{\prime}(0) & 0
\end{array}\right)
$$

and our result is not subsumed under similar known theorems for

Received by the editors September 11, 1958.

1 This research was sponsored by the Office of Ordnance Research, U. S. Army.

2 The prime denotes differentiation with respect to the variable indicated; if no variable is indicated, it denotes differentiation with respect to $t$. 
the existence of a.p. solutions of (1.3) since we allow $g^{\prime}(0)=0$, in which case $L$ has a zero characteristic root.

Biryuk [2] has considered an equation similar to (1.3); namely $u^{\prime}=L u+k F(t, u)$, where $L$ may have a pure imaginary characteristic root. However, he assumes that $F(t, u)=\sum_{n=1}^{N} e^{i \mu_{n} t} F_{\mu_{n}}(u)$, where the $F_{\mu_{n}}(u)$ are polynomials, while we make no other assumption on $e(t)$ than that it be a.p. Moreover, when the perturbation parameter $k=0$, then Biryuk's equation becomes linear, which is not necessarily the case in (1.2) when $k=0$.

Schäffer [3] considers the equation

$$
u^{\prime}+r(u, q(t, k))=0
$$

and uses a perturbation argument about $k=0$. His hypothesis is that

$$
z^{\prime}+\frac{\partial}{\partial u}\left[r\left(u_{0}(t), q(t, 0)\right)\right] z+b(t)=0,
$$

where $u_{0}(t)$ is an a.p. solution of (1.4) when $k=0$, possesses at least one bounded solution for $t \geqq 0$ for every bounded continuous $b(t)$, or at least one bounded solution for $t \leqq 0$ for every such $b(t)$. In our case, the only a.p. solution of (1.2) when $k=0$ is the zero solution, so (1.5) becomes

$$
\begin{aligned}
& z_{1}^{\prime}=z_{2}-f(0) z_{1}+b_{1}(t), \\
& z_{2}^{\prime}=b_{2}(t)
\end{aligned}
$$

when $g^{\prime}(0)=0$. However, if $b_{2}(t)$ is taken to be a nonzero constant, then no solution of (1.6) is bounded, so Schäffer's result is insufficient for the case under consideration.

Amerio [1, pp. 105-119], has shown the existence of an a.p. solution of an equation of the form of (1.3) in which $L$ may have pure imaginary characteristic roots, but not a zero root. The equation he considers is a vector form of the scalar equation

$$
x^{\prime \prime}+\phi\left(x^{\prime}\right)+\gamma x=k e(t)
$$

where $\gamma>0$ is a constant and $\phi$ is strictly increasing but possibly stationary at the origin.

In other known theorems, it is more clearly the case that $L$ in (1.3) has no characteristic roots with zero real parts; e.g., see Reuter [4].

Our proofs are based on the general results of Amerio [1, pp. 97105], who considers the vector equation $u^{\prime}=F(t, u)$, where $F(t, u)$ is a.p. in $t$ and uniformly continuous in $(t, u)$ for $-\infty<t<\infty$ and $u$ in some closed bounded set $\Gamma$ of Euclidean $n$-space. He considers, in fact, the set of equations of the form $u^{\prime}=L(t, u)$, where $L(t, u)$ is 
any member of the closure (taken with respect to the uniform norm over all $u$ in $\Gamma$ and all $t)$ of the set $\{F(t+h, u):-\infty<h<\infty\}$. He then shows that:

(A1). If for some $t_{0}$, the equation $u^{\prime}=F(t, u)$ has a solution $u(t)$ contained in $\Gamma$ for all $t \geqq t_{0}$, then each equation $u^{\prime}=L(t, u)$ has a solution contained in $\Gamma$ for all $t$;

(A2). If each equation of the set $u^{\prime}=L(t, u)$ has a solution $u(t)$ contained in $\Gamma$ for all $t$ which is separated, then each such solution is a.p.; a solution $u(t)$ contained in $\Gamma$ for all $t$ is said to be separated either if it is unique, or if there exists a constant $\rho>0$ such that for any other solution $\bar{u}(t)$ in $\Gamma$ for all $t$,

$$
\inf _{-\infty<t<\infty}|u(t)-\bar{u}(t)| \geqq \rho .
$$

Since our use of these results of Amerio essentially involves the construction of a region $\Gamma$ from which all solutions of our system except one "diverge" as $t \rightarrow-\infty$, but in which all solutions converge as $t \rightarrow+\infty$, the results of Loud [5] are of interest; it is, in fact, observed that our results along that line apply to more general functions $f$ and $g$.

2. We consider the system

$$
\begin{aligned}
& x^{\prime}=y-F(x), \\
& y^{\prime}=-g(x)+k p(t)
\end{aligned}
$$

where $F$ and $g$ are as in (1.2), $k>0$, and $p(t)$ is any element of the closure of the set $\{e(t+h):-\infty<h<\infty\}$. Clearly $|p(t)| \leqq 1$ for all $t$.

We make the following assumptions:

(A) There exist positive numbers $a, b, c, d$, such that $c<d, a\rangle b$, and $g(c)=k, g(-b)=-k$;

(B) For $-a \leqq x \leqq d$ :

(i) $k<(F(d)-F(c)) f(x)+g(-a)$;

(ii) $k<(F(-b)-F(-a)) f(x)-g(d)$;

(iii) $g(x)$ has a continuous derivative $g^{\prime}(x), g(0)=0$, and $0<g^{\prime}(x)$ $\leqq \beta$ for $x \neq 0$;

(iv) $f(x) \geqq \alpha>0$;

(v) $\beta<\alpha^{2}$.

These conditions, for example, are satisfied if (iii), (iv), and (v) hold for all $x$, and in addition,

(vi) $\lim \inf _{|x| \rightarrow \infty}|g(x)|>k$.

For then there clearly exist $c>0, b>0$, such that $g(c)=k, g(-b)=-k$, and we simply take

(vii) $a>\left(\left(\alpha^{2}+\beta\right) /\left(\alpha^{2}-\beta\right)\right) \max (b, c)$, and $d=a$. 
Hence

$$
\begin{aligned}
k & =g(c)=g(-a)+(g(c)-g(-a)) \\
& \leqq g(-a)+\beta(c+d) \\
& =g(-a)+\alpha^{2}(d-c)-\left(\alpha^{2}-\beta\right)\left(d-\frac{\alpha^{2}+\beta}{\alpha^{2}-\beta} c\right) \\
& <g(-a)+\alpha^{2}(d-c) .
\end{aligned}
$$

But $\alpha(d-c) \leqq F(d)-F(c)$, so

$$
\begin{aligned}
k & <g(-a)+(F(d)-F(c)) \alpha \\
& \leqq g(-a)+(F(d)-F(c)) f(x),
\end{aligned}
$$

and (i) is satisfied. Similarly (ii) can be shown to hold; we omit the details.

If (iii) and (iv) hold in some interval $I$ containing the origin, and there exist positive constants $M$ and $\gamma$ such that

$$
\text { (viii) }|g(x)| \leqq M|x|^{\gamma+1}
$$

for all $x \in I$, then for fixed $\alpha$ and $k$ sufficiently small, conditions (A) and (B) can again be realized. Indeed, since $g(0)=0$, it follows that for $k \rightarrow 0$, we have $b \rightarrow 0$ and $c \rightarrow 0$, where $b$ and $c$ are as determined in (A). But (viii) clearly implies $g^{\prime}(0)=0$. Thus if $a=d=\max (2 b, 2 c)$, then $k \rightarrow 0$ implies $\beta \rightarrow 0$, where $\beta$ is as determined in (iii).

Hence, for $k$ sufficiently small, $\beta<\alpha^{2}$ and

$$
\frac{\alpha^{2}+\beta}{\alpha^{2}-\beta}<2
$$

Thus, clearly, conditions (v) and (vii) hold, and therefore conditions (A) and (B) are again realized.

Definition. A relative bound for (2.1) is a bounded region $R$ of the $(x, y)$ plane such that if for any $t_{0}$, and any solution $(x(t), y(t))$ of (2.1), we have $\left(x\left(t_{0}\right), y\left(t_{0}\right)\right) \in R$, then $(x(t), y(t)) \in R$ for all $t>t_{0}$.

We now construct a relative bound for (2.1). Put $\beta_{1}=F(d)-F(c)$ and $\beta_{2}=F(-b)-F(-a)$, and let $R$ be the bounded region bounded by the closed curve consisting of the following arcs:

$$
\begin{array}{rlrl}
\Gamma_{1}: y & =F(x)+\beta_{1}, & -a & \leqq x \leqq c ; \\
\Gamma_{2}: y & =F(d), \quad F(d)-\beta_{2} & \leqq y \leqq F(d) ; \\
\Gamma_{3}: x & =d, \quad-b \leqq x \leqq d ; \\
\Gamma_{4}: y & =F(x)-\beta_{2}, \quad-b \\
\Gamma_{5}: y & =F(-a), & -a & \leqq x \leqq-b ; \\
\Gamma_{6}: x & =-a, & F(-a) & \leqq y \leqq F(-a)+\beta_{1} .
\end{array}
$$


Lemma 2.1. The region $R$ as defined above is a relative bound for (2.1).

Proof. At a point of $\Gamma_{1}$ for which $-a \leqq x<c$, a trajectory of (2.1) has a slope given by

$$
\frac{y^{\prime}}{x^{\prime}}=\frac{-g(x)+k p(t)}{\beta_{1}} \leqq \frac{-g(-a)+k}{\beta_{1}} ;
$$

by (i) it thus follows that

$$
\frac{y^{\prime}}{x^{\prime}}<f(x)=F^{\prime}(x)
$$

and hence this slope is less than that of $\Gamma_{1}$ at the point. Since also $x^{\prime}=\beta_{1}>0$ on $\Gamma_{1}$, it follows that the trajectory enters $R$ as $t$ increases.

At a point of $\Gamma_{2}$ such that $c<x<d$, we have $y^{\prime}=-g(x)+k p(t)$ $<-g(c)+k=0$; hence the trajectories of (2.1) cross this segment so as to enter $R$ as $t$ increases. A similar statement applies to trajectories containing points on $\Gamma_{3}$ for which $F(d)-\beta_{2} \leqq y<F(d)$, since here $x^{\prime}=y-F(x)<0$.

At the point $(d, F(d)), x^{\prime}=0, y^{\prime}<0$. Hence any trajectory at this point is tangent to the line $x=d$ and moves toward the $x$-axis as $t$ increases. Just below this point, $y<F(x)$, so such a trajectory must then satisfy $x^{\prime}<0$ and thus enter $R$ as $t$ increases.

At the point $(c, F(d)), y^{\prime}=-g(c)+k p(t) \leqq 0$ and $x^{\prime}>0$; hence, any trajectory there moves into $R$ or is tangent to $y=F(d)$. If the latter case holds, then since the trajectory moves to the right as $t$ increases, $y^{\prime}$ becomes negative, and the trajectory must also move toward the $x$-axis, i.e., enter $R$ as $t$ increases.

Since other parts of the boundary of $R$ have essentially the same properties with respect to the trajectories of (2.1), we omit the rest of the proof of the lemma.

It follows by (A1), the first of the two general results due to Amerio, that (2.1) has a solution $(\bar{x}(t), \bar{y}(t)) \in R$ for all $t$. We show next that this solution is unique; i.e., separated; it will then follow from (A2), the second general result of Amerio, that this solution is a.p.

Suppose $(x(t), y(t)) \in R$ for all $t$ but $(x(t), y(t)) \neq(\bar{x}(t), \bar{y}(t))$. Put $\xi(t)=x(t)-\bar{x}(t), \eta(t)=y(t)-\bar{y}(t)$. Clearly

$$
\begin{aligned}
\xi^{\prime} & =\eta-m(\bar{x}, \xi) \xi, \\
\eta^{\prime} & =-h(\bar{x}, \xi) \xi
\end{aligned}
$$

where $\xi=\xi(t), \eta=\eta(t), \bar{x}=\bar{x}(t)$, 


$$
m(\bar{x}, \xi)=\left\{\begin{array}{cc}
(F(\bar{x}+\xi)-F(\bar{x})) / \xi, & \xi \neq 0, \\
f(\bar{x}), & \xi=0
\end{array}\right.
$$

and

$$
h(\bar{x}, \xi)=\left\{\begin{array}{cc}
(g(\bar{x}+\xi)-g(\bar{x})) / \xi, & \xi \neq 0, \\
g^{\prime}(\bar{x}), & \xi=0 .
\end{array}\right.
$$

Since $-a \leqq \bar{x}(t) \leqq d$ and $-a \leqq \bar{x}(t)+\xi(t) \leqq d$, there exists a constant $M>0$ such that for all $t$,

$$
0<\alpha \leqq m(\bar{x}, \xi) \leqq M .
$$

We now construct a "length" function on the vectors $(\xi, \eta)$ and, to this end prove the following

Lemma 2.2. If $0<\alpha \leqq m(t) \leqq M$, then the Riccati equation

$$
A^{\prime}=(m(t)-A) A
$$

has a solution $A(t)$ such that $\alpha \leqq A(t) \leqq M$ for all $t .^{3}$

Proof. Let $A\left(t, A_{0}\right)$ be the solution of (2.5) such that $A\left(0, A_{0}\right)=A_{0}$. It is clear that if $A_{0}>M$, then $A\left(t, A_{0}\right)>M$ for $t<0$. It is also easy to show that if $0<A_{0}<\alpha$, then $A\left(t, A_{0}\right) \rightarrow 0$ as $t \rightarrow-\infty$, while $A\left(t, A_{0}\right)$ $>0$ for $t<0$; we omit the details.

Now consider the set of positive numbers $A_{0}$ such that $A\left(t, A_{0}\right) \rightarrow 0$ as $t \rightarrow-\infty$. Since this set is nonvoid and bounded, it has a least upper bound $A_{1}$. It is also clear that this set $S$ is an interval, for if $A_{0} \in S$, and $0<\bar{A}_{0}<A_{0}$, then $\bar{A}_{0} \in S$, since the graphs of $A=A\left(t, A_{0}\right)$ and $A=A\left(t, \bar{A}_{0}\right)$ cannot intersect. Suppose now that $A\left(t_{1}, A_{1}\right)<\alpha$ for some $t_{1}<0$; then by the continuity of solutions as functions of initial conditions, it follows that for some $A_{2}>A_{1}, A\left(t_{1}, A_{2}\right)<\alpha$. But then, clearly $A\left(t, A_{2}\right) \rightarrow 0$ as $t \rightarrow-\infty$; i.e., $A_{2} \in S$ which is a contradiction. Hence $A\left(t, A_{1}\right) \geqq \alpha$ for $t<0$.

If $A\left(t_{1}, A_{1}\right)>M$ for some $t_{1}<0$, a similar continuity argument shows that for some $A_{0}<A_{1}, A\left(t, A_{0}\right)>M$ for $t<t_{1}$; since $A_{0} \in S$ this is again a contradiction. Hence $\alpha \leqq A\left(t, A_{1}\right) \leqq M$ for $t<0$, and this inequality clearly also holds at $t=0$.

Clearly for any solution of (2.5), $A(t)>M$ implies $A^{\prime}<0$ and $A(t)<\alpha$ implies $A^{\prime}(t)>0$. Hence if the solution $A\left(t, A_{1}\right)$ leaves the strip $\alpha \leqq A \leqq M$ for $t>0$, then $A\left(t, A_{1}\right)$ must have a positive relative maximum or minimum outside this strip at some $t_{2}>0$. But $A^{\prime}\left(t_{2}, A_{1}\right)$

${ }^{3}$ This may be a well known result since it can also be proved by considering the explicit general solution of the Riccati equation. 
$=0$; i.e., $A\left(t_{2}, A_{1}\right)=m\left(t_{2}\right)$; but $\alpha \leqq m\left(t_{2}\right) \leqq M$, and we have a contradiction. This completes the proof of the lemma.

We now take $m(t)=m(\bar{x}(t), \xi(t))$ as defined in (2.3), denote by $A(t)$ the solution $A\left(t, A_{1}\right)$ of (2.5) assured by Lemma 2.2, and define the "length"

$$
D=\left(\eta^{2}+(\eta-A(t) \xi)^{2}\right)^{1 / 2} .
$$

From (2.2) and (2.5) we have for $\xi=\xi(t), \eta=\eta(t), A=A(t)$ :

$$
D D^{\prime}=-P \xi^{2}+2 Q \xi \eta-A \eta^{2}
$$

where

$$
P=A\left(A^{2}-h\right), \quad Q=A^{2}-h,
$$

and

$$
Q^{2}-P A=-h\left(A^{2}-h\right) .
$$

Lemma 2.3. If $(\xi, \eta) \neq(0,0)$, then $D^{\prime}<0$.

Proof. By (iii), (v), (2.4), and the fact that $A(t) \geqq \alpha$ for all $t$, we have $Q^{2}-P A=-h\left(A^{2}-h\right) \leqq 0$ with the equality possible only if $h=0$; i.e., if $\xi=0$. If $\xi=0$ then $D D^{\prime}=-A \eta^{2}<0$ unless also $\eta=0$. Hence $D D^{\prime}<0$ for $(\xi, \eta) \neq(0,0)$ and since also $D>0$, the lemma follows.

Lemma 2.4. The solution $(\bar{x}(t), \bar{y}(t)) \in R$ for all $t$ is unique.

Proof. If the solution $(\bar{x}(t), \bar{y}(t))$ is not unique, then $(\xi(t), \eta(t))$ $\neq(0,0)$ for all $t$, since if $\left(\xi\left(t_{1}\right), \eta\left(t_{1}\right)\right)=(0,0)$, then $(\xi(t), \eta(t))=(0,0)$ for all $t$ by the uniqueness of solutions of (2.2).

Hence $D>0$, and by Lemma $2.3, D^{2}$ is a strictly decreasing function of $t$. Moreover, since $\xi(t), \eta(t)$, and $A(t)$ are bounded for all $t, D^{2}$ is also, and $D^{2} \rightarrow D_{0}^{2}$ as $t \rightarrow-\infty, 0<D_{0}<\infty$. This implies $D D^{\prime} \rightarrow 0$ on some sequence of $t$ 's tending to $-\infty$. From (2.7),

$$
D D^{\prime}=-A\left[\left(\eta-\frac{Q}{A} \xi\right)^{2}+\left(\frac{P A-Q^{2}}{A^{2}}\right) \xi^{2}\right],
$$

and since $0<\alpha \leqq A$ and $P A-Q^{2} \geqq 0$, then either $\xi \rightarrow 0$ or $P A-Q^{2} \rightarrow 0$ on this sequence. The second possibility implies that $h \rightarrow 0$ on this sequence, since in (2.9) $A^{2}-h \geqq \alpha^{2}-\beta>0$ by (v). If $\xi \rightarrow 0$ on this sequence, then there is a subsequence on which $\xi \rightarrow \xi_{0} \neq 0$ and $\bar{x} \rightarrow \bar{x}_{0}$. Since $h$ is continuous in $(\bar{x}, \xi)$, it follows that $h(\bar{x}, \xi) \rightarrow h\left(\bar{x}_{0}, \xi_{0}\right)$ on this subsequence. But $\xi_{0} \neq 0$ implies $h\left(\bar{x}_{0}, \xi_{0}\right)>0$, contradicting the fact that $h \rightarrow 0$. Hence in any case $\xi \rightarrow 0$ on a sequence of $t$ 's tending to 
$-\infty$, on which, of course, $D D^{\prime} \rightarrow 0$ also. From (2.7), then also $\eta \rightarrow 0$ and from (2.6), $D \rightarrow 0$. This contradicts $D \rightarrow D_{0}>0$, and the proof is complete.

As a consequence of these lemmas, and Amerio's result (A2) we have the following

THEOREM. Under assumptions (A) and (B), there exists an a.p. solution $(\bar{x}(t), \bar{y}(t))$ of (2.1). The trajectory of this solution is contained in the region $R$ of Lemma 2.1 and this solution is asymptotically stable with respect to all solutions whose trajectories enter $R$.

Proof. Only the last part of the second assertion remains to be established, but this follows by an argument similar to that in the proof of Lemma 2.4. In fact, any other solution $(x(t), y(t))$ in $R$ at $t=t_{0}$ remains in $R$ for $t>t_{0}$. Hence if $\xi, \eta$, and $D$ are as defined before, we have $D D^{\prime}<0$ again for $t>t_{0}$. But $D^{2} \rightarrow D_{1}^{2}$ as $t \rightarrow+\infty$, and the assumption $D_{1}>0$ leads again to a contradiction. Hence $D^{2} \rightarrow 0$ as $t \rightarrow+\infty$ and since $A(t) \geqq \alpha>0$ then $D^{2} \rightarrow 0$ implies $(\xi, \eta) \rightarrow(0,0)$. This proves the theorem.

\section{REFERENCES}

1. L. Amerio, Soluzioni quasi-periodiche, o limitate, di sistemi differenziali non lineari quasi-periodici, o limitati, Ann. Mat. Pura Appl. vol. 39 (1955) pp. 97-119.

2. G. I. Biryuk, On the existence of almost periodic solutions of nonlinear systems with a small parameter in the case of degeneration, Dokl. Akad. Nauk. SSSR (N.S.) vol. 97 (1954) pp. 577-579.

3. J. J. Schäffer, Analytische Parameterabhängigkeit der fastperiodische Lösungen von nichtlinearen Differentialgleichungen, Rend. Circ. Mat. Palermo (2) vol. 5 (1956) pp. 204-236.

4. G. E. H. Reuter, On certain nonlinear differential equations with almost periodic solutions, J. London Math. Soc. vol. 26 (1951) pp. 215-221.

5. W. S. Loud, Boundedness and convergence of solutions of $x^{\prime \prime}+c x^{\prime}+g(x)=e(t)$, Duke Math. J. vol. 24 (1), (1957) pp. 63-72.

Iowa State College of Agriculture and Mechanic Arts 